\title{
EI "Informe Sobre Ciegos" y Fernando Vidal Olmos, Poeta Vidente
}

Is this not tse essence of the modern belief about the nature of the artist, the man who goes down into that hell which is the bistorical beginning of the buman soul, a beginning not outgrown but establisbed in bumanity as we know it now, preferring the reality of this hell to the bland lies of the civilization that bas overlaid it?

Lionel Trilling, Beyond Culture

Je dis qu'il faut être Voyant, se faire Voyant

Arthur Rimbaud

Uno de los puntales del pensamiento de Ernesto Sábato - presente en sus ensayos y objetivado en su ficción- lo constituye su actitud de rechazo hacia los valores de Occidente, valores que, al convertir a la razón en deidad suprema y al sofocar lo instintivo-elemental del hombre, han convertido a éste en un ser inauténtico, angustiado y solitário. La llamada civilización moderna ha enajenado a la criatura humana, la ha enajenado de sí misma y también del universo y la integración original hombre-cosmos ha sido así desgarrada. De ahí la angustia del hombre, de ahí su soledad. ${ }^{1}$

Sabre béroes y tumbas representa un ataque directo a todo tipo de conformismo burgués idiotizante, epitome de nuestra civilización. Sábato ha partido de una realidad geográfica e histórica concreta -Argentina

1. El rechazo de la civilización ha sido reconocido como un lugar común de la literatura moderna: "It seems to me that the characteristic element of modern literature, or at least of the most highly developed modern literature, is the bitter line of hostility to civilization which runs through it," Lionel Trilling, Beyond Culture (New York, 1968), p. 3. 
y Buenos Aires - pero la ha trascendido universalizándola. En rigor, se trata de un enjuiciamiento a toda una civilización, a toda una concepción racionalista de la vida y a un mal interpretado progreso cuyo símbolo es, precisamente, la ciudad, en este caso la babilónica Buenos Aires. Es por eso que uno de los personajes centrales, Fernando Vidal Olmos, un perfecto canalla, parece imbuido de una gran pureza en contraste con la sociedad que él denigra; rebelde y loco, ha conservado su autenticidad de ser humano junto a seres despreciables, pilares de la comunidad, grandes hipócritas que mueven el mundo. Fernando, tal como aparece en el "Informe sobre ciegos," se convierte en símbolo del hombre contemporáneo, un ser que se desgarra angustiado entre los dos males que conforman su existir; por un lado, lo primario-animal de la naturaleza humana que persiste en el fondo del ser y que en nombre de la razón rechaza; por el otro, el paraíso artificial -civilización, cultura-- que él mismo ha erigido y que, de algún modo, lo engrandece pero que inevitablemente lo destruye al sofocar su esencia. Es así que el hombre se ha convertido en "ese ser dual y desgraciado que se mueve y vive entre la tierra de los animales y el cielo de sus dioses, que habrá perdido el paraíso terrenal de su inocencia y no habrá ganado el paraíso celeste de su redención". ${ }^{3}$ Para Sábato, el ser humano ha pagado caro la gran aventura humanista de la modernidad iniciada en el Renacimiento ya que ésta ha culminado en su propia deshumanización o cosificación; el

\footnotetext{
a Más que al género novelístico el "Informe sobre ciegos" se ajusta a la forma narrativa que Northrop Ftye ha definido como romance: "The essencial difference between novel and romance lies in the conception of characterization. The romancer does not attempt to create 'real people' so much as stylized figures which expand into psychological archetypes." (Northrop Frye, Anatomy of Criticism, New York, 1966), p. 304. También aclara Frye que mientras el novelista, "deals with personality, with characters wearing their personse or social masks... The romancer deals with individuality, with characters in vacuo, idealized by revery." (p. 305) Sacado del marco histórico de la ciudad de Buenos Aires $y$ arrojado en un universo intemporal, Fernando Vidal se convierte en la figura arquetípica del Hombre que busca su salvación.

3 Ernesto Sábato, Sobre héroes y tumbas (Buenos Aires, 1969), pp. 432-3. Todas las citas subsiguientes serán de esta edición.

4 Angela Dellepiane ha estudiado el influjo que sobre Sábato han ejercido varios escritores tan preocupados como él por la deshumanización del hombre contemporáneo. Escribe la autora: "En esta etapa de su ensayística (la de $E l$ escritor $y$ sus fantasmas) el influjo de las ideas de Lewis Mumford $y$ Erick Kahler, junto con las de Berdiaeff... es lo más significativo en la formación de las opiniones de Sábato. Con el primero compartirá ideas tales como la de que la humanización de la máquina ha tenido el paradójico efecto de mecanizar a 12 humanidad... Con Berdiaeff, Sábato coincide en opinar que la tragedia, colectiva e individual, del hombre reside en su 'cosificación' ('objectification' es la palabra en el existencialista cristiano), que es una degradación y pérdida even. tual del impulso creador... De Kahler, Sábato ha absorbico las ideas acerca del significado de la Historia: del Renacimiento, como la época en que el hombre se libera $y$ progresa ilimitadamente, basado en el poder de la razón. Kahler ve
} 
desgarramiento y la angustia on que vive constituyen, "el final contra. dictorio de aquel semidiós que proclamó su individualidad en los albores del Renacimiento, de aquel ser que se lanzó a la conquista de las cosas" y que "ignoraba que él mismo sería convertido en cosa." s Sábato, sin embargo, no se contenta con enjuiciar a la sociedad contemporánea: Sobre béroes y tumbas en general y el "Informe sobre ciegos" en particular proponen una posible salida del laberinto de la civilización tecno. látrica. Para ello es menester que el hombre reconsidere los valores por los que se ha guiado durante siglos y que, sin abandonar las viejas normas, sepa integrarlas en un esquema más amplio. "Vivimos el momento en que es necesaria una nueva síntesis", ha declarado Sábato, una sín. tesis en la que la razón se integre a las fuerzas ocultas e irracionales que inevitablemente rigen nuestro universo $y$ moldean nuestras vidas. Es necesario trascender la materia que esclaviza y dar el gran salto hacia una realidad más vasta y hacia la plenitud del ser.

En rigor, las fuerzas irracionales e instintivas constituyen para Sá. bato el lado más genuino y revelador del ser: la salvación del hombre concreto que el autor propone depende, por lo tanto, del retorno de éste a su unidad primigenia - razón e instinto- después de una inmersión necesaria o viaje al fondo de su conciencia, retorno que implica al mismo tiempo una reintegración del ser a la unidad primordial hombre. cosmos. Para Sábato, la novela, más que otra manifestación artística, está destinada a ser el medio para dar cuenta de ese rescate del hombre concreto e integral, por ser la ficción género híbrido que registra e indaga lo consciente y lo inconsciente, el pensamiento y la fantasía, lo diurno y lo nocturno tanto del individuo como de la humanidad. ${ }^{7}$ De modo que, de entre todos los hombres, sólo el artista - en este caso particularmente el novelista- es capaz de dar cuenta de esa visión más amplia de la realidad; el novelista baja, como Orfeo, a su "yo" profundo y allí descubre un mundo mágico, primitivo y mitológico que es,

al hombre actual trabado por relaciones que ni comprende ni controla, atormentado y temeroso." (Angela Dellepiane, Emesto Sábato: el bombre y su obra, New York, 1968, pp. 167-8).

5 Ernesto Sábato, El escritor y sus fantasmas (Buenos Aires, 1967), p. 55. Todas las citas subsiguientes serán de esta edición. Leemos en otro lugar: "El Renacimiento produjo tres paradojas: fue un movimiento individualista que condujo a la masificación; fue un movimiento naturalista que terminó en la máquina; $y$, en fin, fue un humanismo que desembocó en la deshumanización". (p. 55).

- Ernesto Sábato, Hombres y engranajes (Buenos Aires, 1970), p. 108.

7 Ha declarado Sábato: "En tanto que la novela, por ser un género híbrido, por participar a la vez del mundo luminoso y del mundo oscuro, del mundos mental y del mundo instintivo, del mundo racional y del mundo irracional, puede dar, y está dando en realidad, la gran síntesis". Emir Rodríguez Monegal, $E$, arte de narras (Caracas, 1968), p. 250. 
a la vez, patrimonio de toda la humanidad. Al mismo tiempo, un viaje al fondo de la conciencia equivale a un descenso -o retorno- a "las regiones inmemoriales de la raza, alli donde dominan los instintos bá. sicos de la vida y de la muerte, donde el sexo y el incesto, la paternidad y el parricidio mueven sus fantasmas". 8 Ahora bien, Fernando, además de ser personaje de Sobre béroes y tumbas, encarna la concepción sabar tiana del artista, es decir, "el único por excelencia... el que gracias a su incapacidad de adaptación, a su rebeldía, a su locura, ha conservado paradojalmente los artibutos más preciosos del ser humano."9 El Informe es su propia creación poética y en él da cuenta de su rebeldía y de su visión: este gran inadaptado se torna una figura satánica al volverse contra una sociedad de hombre-cosas cuyo más genuino representante está encarnado en la grotesca figura de Molinari. En suma, si Sobre béroes y tumbas y el Informe proponen una posible salvación del hombre concreto, esclavo como es de una civilización superracionalista y deshumanizada, es precisamente Fernando, por ser artista, quien está destinado a efectuar tal rescate. Al rescatar la plenitud de su ser también recobra, de alguna manera, la integridad del ser de los demás: por medio de su obra poética, Fernando les infarma y les hace partícipes de su aventura metafísica.

Si bien es cierto que la figura del artista, tal como Sábato la concibe, abarca los grandes visionarios de todos los tiempos, también es verdad que la arrogancia satánica y destructora de Fernando lo identifican específicamente con los poetas llamados malditos, de la talla de Rimbaud y Lautréamont; es decir, con aquellos "profetas prematuros del desastre" cuyos mensajes "flotaron en el vasto océano del siglo XIX, hasta que pudieron ser hallados y justicieramente interpretados".10 $\mathrm{Al}$ mismo tiempo, Fernando encarna al héroe surrealista, al digno heredero de la tradición maldita; no sólo persigue y logra la realidad absoluta por la que abogaron los del grupo de André Breton, sino que su actitud vital puede resumirse en un desesperado anhelo por transformar la rea. lidad trascendiéndola, transmutándola. ${ }^{11}$

8 Ernesto Sábato, El escritor y sus fantasmas, p. 263.

- Ibid., p. 74 .

10 Ibid., p. 68.

11 Hemos dicho que Fernando Vidal en cuanto personaje de Sobre béroes y tumbas representa al hombre contemporáneo, a ese ser enajenado que con el tiempo se ha convertido en mero engranaje de la maquinaria del progreso. Fernando, el Artista, por el contrario, está situado en un contexto histórico mucho más vasto; en última instancia encarna una concepción del poeta que es una constante en la historia de la cultura occidental desde Platón y que adquiere vigencia nuevamente con el romanticismo, sobre todo con el romanticismo alemán: el poeta como poseso, como vate. 
La relación existente entre Fernando Vidal y su Informe es paralela a la de Sábato y Sobre béroes y tumbas. Ambos han partido de una realidad concreta y objetiva pero la han transmutado haciendo de sus res. pectivas visiones finales perfectas síntesis donde la realidad y el sueño, lo cotidiano y lo fantástico han dejado de ser percibidos como antinomias para resolverse en una sola entidad. En verdad, y como lo ha admitido Sábato, el Informe es para Sobre béroes y tumbas lo que los sueños $y$ visiones son en la vida de un hombre, lo que las alucinaciones $y$ pesadillas son en la vida de Fernando. Ha declarado el autor: "en mi novela pretendí dar la realidad en toda su extensión y profundidad, incluyendo no sólo la parte diurna de la existencia sino la parte nocturna y tenebrosa". ${ }^{12}$ Ahora bien, la realidad así objetivada apunta a la noción de suprarrealidad o superrealidad, la meta anhelada del grupo encabezado por Bretón. Leemos en el Primer Manifiesto del Surrealismo: "Yo creo firmemente en la fusión futura de esos dos estados, aparentemente tan contradictorios: el sueño y la realidad, en una especie de realidad absoluta, de superrealidad"; ${ }^{13}$ vale decir, un orden superior existente más allá del universo sensible y que ilumina este mundo precario de opuestos y de contradicciones. Sábato ha adoptado él mismo la consigna de los surrealistas para quienes el Surrealismo implica no sólo un credo poé.

En el marco de Sobre béroes y tumbas, sin embargo, Fernando representa, en primer lugar, al ideal del poeta y héroe surrealista; esto es cierto sobre todo en lo que se refiere al logro de una realidad absoluta y en lo que atañe al papel que el azar objetivo juega en la conformación de su destino. Esta afiliación con el surrealismo se presenta, además, entrañablemente identificada con la tradición de los llamados poetas malditos del siglo XIX, malditos pues ellos en la realidad, como Fernando en la ficción, han sido los receptores de verdades que el común de los mortales no podría tolerar. Sobre el calificativo de maldito leemos en la Encyclopedia of Poetry and Poetics: "It was given currency by Verlaine's Les poètes maudits (1884-1888), a collection of essays on poets hardly known at the time, such as Corbière, Rimbaud and Mallarmé. $A$ half-century earlier Vigny's Stello (1832) had developed. $\because$ the idea that poets... are envied and hated for their qualities by society and its rulers who fear the truths they tell". (Alex Preminger, Encyclopedia of Poetry and Poetics, Princeton 1965, p. 626). No tiene nada de extraño, por lo tanto, que escritores como Rimbaud, Lautréamont y Strindberg, también visitadores del infierno, sean mencionados repetidas veces en el Informe.

Finalmente, la experiencia de Fernando, verdadero viaje espiritual que culmina con la adquisición de la videncia, puede equipararse con la visión del poeta tal como la enuncia Rimbaud en su "Lettre dite du voyant" dirigida a Paul Demeny en 1871: "La première étude de l'homme qui veut être poète est sa propre conaissance entière; il cherche son âme, il l'inspecte, il la tente, l'apprend. Dès qu'il la sait, il doit la cultiver. . Mais il s'agit de faire l'âme monstrueuse... Je dis qu'il faut être Voyant, se faire Voyant. Le Poète se fait Voyant par un long. immense et raisonné dérèglement de tous les sens... Ineffable torture... où il devient entre nous le grand malade, le grand criminel, le gran maudit, -et le Suprême Savant! - Car il arrive à l'inconnu." (Editions Messein, Paris, 1954).

12 Ernesto Sábato, El escritoy y sus fantasmas, p. 19.

13 André Breton, Los manifiestos del surrealismo (Buenos Aires, 1965), p. 29. 
tico sino también una actitud ante la vida. Esta se resuelve en una incansable búsqueda de autenticidad que permitirá al ser humanizarse, rescatarse de su condición de cosa. Y si el artista encarna para Sábato al hombre rescatado por excelencia, el "Informe sobre ciegos", expresión poética de una formidable experiencia vital, se convierte en testimonio de ese rescate. De hecho, el Informe está construido alrededor de dos ejes temáticos de índole surrealista que mutuamente se implican: la ya mencionada búsqueda de una realidad absoluta y la visión final de un orden en el que hombre y cosmos han dejado de percibirse como entidades contradictorias.

\section{1) BÚSQUEdA DE UNA REALIDAd ABSOLUTA}

El Informe queda claramente dividido en dos secciones o momentos: la etapa preparatoria, investigación o búsqueda, y la entrada en el universo maldito simbolizada por la irrupción del protagonista en la casa de Belgrano, fin de su exploración y culminación de su experiencia. A lo largo del Informe, sin embargo, la divisa es una sola: conquistar el infinito, alcanzar el absoluto investigando el mal encarnado en la Secta de los Ciegos. El símbolo, un viaje; un viaje a la eternidad sin desplazamiento y sin tiempo. Después de invocar a los dioses de las tinieblas inicia Fernando su descenso al infierno -al suyo propio $y$ al infierno de la humanidad- a la vez que comienza a dar expresión poética a su íntima experiencia. Como otros antes - Rimbaud, Lautréar mont, Maupassant, Artaud- Fernando, poeta en la ficción, se sabe destinado a penetrar el sentido secreto de la realidad y a informar al mundo de la verdad antes de sucumbir a la etapa final de su destino.

En su aspecto inmediato el Informe refiere la historia de las investigaciones que, sobre las fuerzas del mal, ha realizado Fernando Vidal Olmos: una consciente y sistemática y la otra inconsciente, llevadas a cabo desde las épocas más remotas de la infancia del protagonista. Fernando encarna así al héroe surtealista que mira y ve más allá de lo concreto y de lo aparencial: "Y así fui advirtiendo detrás de las apar riencias el mundo abominable"; $;^{14}$ de esta manera le es permitido vis. lumbrar poco a poco la verdad y percibirla aun en los hechos cotidianos más banales como son las pesadillas y los sueños. Su perspectiva se resuelve en una infinita expansión de la realidad ya que el protagonista anhela develar un más allá inmanente en la realidad misma: de ani que

14 Sobre béroes, p. 35. 
su Informe proporcione una visión final en donde lo real ha sido no simplemente trascendido sino transmutado, transfigurado. Esto es lo que Anna Balakian ha designado como la revelación de la presencia del infinito en la materia, "the revelation of the presence of the infinite in matter", para luego añadir, "(the revelation) is considered a subjective act of exploration rather than a denial of the limitations of objective reality: the dominant symbol of this exploration is the familiar one of the voyage, but a voyage totally transformed." 15 Todo el Informe es la objetivación de una surrealidad; en él se borran las fronteras entre el mundo objetivo exterior y el subjetivo, desaparece la línea divisoria que separa los estados de sueño y de vigilia, de locura y de sensatez. En el Informe son precisamente esos estados misteriosos de locura y pesadilla los que causan en el personaje una mayor comprensión de su realidad interna a la par que le revelan nuevos e insospechados aspectos de una verdad externa más amplia y más rica, aunque, claro, más tenebrosa y mortal.

Una campanilla de ciego en un rincón de Buenos Aires ha despettado a Fernando para siempre de ese sueño que otros seguirán soñando: "Delante de mí... vi a la ciega. . Había cesado de tocar su campanilla; como si sólo la hubiese movido para mí, para despertarme de mi insen. sato sueño, para advertir que mi existencia anterior había terminado, como una estúpida etapa preparatoria, y que ahora debía enfrentarme con la realidad". ${ }^{16}$ Estas palabras prefiguran la iluminación final que constituye la culminación de la experiencia del protagonista y que se resuelve en el logro del conocimiento supremo: Fernando es el poseído, el vidente, el poeta maldito cuyo destino es imposible soslayar

Los periodos alucinatorios que registran la historia del héroe constituyen un todo coherente y en el contexto del Informe representan lo que éste en el de Sabre béroes y umbas: el lado nocturno y alucinante de la realidad. ${ }^{17}$ La primera visión, la de la gruta, marca el comienzo de la experiencia enajenante que culminará con Ia toma de conciencia final: Fernando entra en aquel recinto identificado por fin con el mal que investigaba y solamente entonces, ya ciego —egado- podrá ver la verdad. ${ }^{18}$

15 Anna Balakian, The Literary Origins of Surrealism (New York, 1957), p. 100 .

16 Sobre béroes, p. 236.

17 Es claro que el "Informe sobre ciegos" puede también ser considerado como una extendida alucinación.

18 A esta visión primera suceden una serie de recuerdos: reminiscencias de trágicas historias sobre ciegos, evocaciones de países visitados, tenebrosas experiencias con una modelo ciega, el encuentro con el pintor Domínguez y finalmente 
Las visiones finales - cloacas, cavernas, torres deidad - apuntan a la etapa última del descenso a los infiernos. Y si el abandonado departamento adyacente a la iglesia simboliza la entrada en el universo de los ciegos, las cloacas de Buenos Aires evocan la visión de un descenso al principio de la raza y del propio ser. Allí, en los orígenes, Fernando, solitario y poderoso, descubre que ha empezado, por fin, a comprender el misterio central de la existencia. Esta visión descendente final da realidad artística al momento crucial de la experiencia del héroe, momento en que el ego del protagonista se disuelve en el ser colectivo de la raza - el inconsciente colectivo de Jung- para identificarse con el eterno espíritu del mal. De esta visión última tesalta el momento supremo, el del incesto, la tenebrosa cópula que representa la inevitable alianza del alma del poeta con los poderes infernales que dominan el mundo: "ver finalmente las grandes fuerzas de las tinieblas como los místicos alcanzan a ver al dios de la luz y de la bondad. $Y$ yo, místico de la Basura y del Infierno puedo y debo decir: CREED EN Mí!"19 Y así Fernando se sume aparentemente en la abrumadora y absoluta nada; a punto de entrar en el vientre de la deidad, el protagonista comprende que todo aquello - las torres, la desolada comarca, el recinto de la deidad, el astro declinante- habían estado esperando su llegada y que sólo por eso no se habían derrumbado hacia la nada, que una vez que lograra penetrar en el Ojo Fosforescente todo se desvanecería como un simulacro milenario. El mismo desaparece, metamorfoseado, convertido en pez antes de disolverse en el maligno vientre. $\mathrm{Y}$ allí, en el centro, en el instante mismo de la enajenación total, Fernando vive esa experiencia que ha sido designada como el supremo momento surrealista, es decir "that moment when the poet arrives at the center of himself and, therefore, at the center of human destiny, when he participates in the consciousness of the world and there establishes a point of contact between himself and the world." ${ }^{20}$ La experiencia de Fernando es,

la mención de todo el grupo surrealista encabezado por Bretón; la inserción de esta serie de episodios -aparentemente reales en la existencia diurna del protagonista- entre un sinnúmero de pesadillas y visiones contribuye a crear un ejemplo de la realidad absoluta por la que abogaron Bretón y sus seguidores. No es extraño entonces que en el Informe los conocidos héroes surrealistas que Sábato frecuentó estén presentados como presidiéndola y que, a la vez, los veamos desrealizados, convertidos en personajes de esta suprarrealidad.

19 Sobre béroes, p. 353.

20 Wallace Fowlie, Age of Surrealism (Bloomington, Indiana, 1966), pp. 26-27. Por su parte el autor francés Michel Carrouges emplea la noción de punto supremo para designar este momento cumbre de la aventura surrealista a la vez que llama la atención sobre su origen esotérico: "La notion de point suprême est la pierre d'angle fondamentale de la cosmologie surréaliste, elle est le foyer vivant du réel et du surréel et elleve vient de l'esotérisme." (André 
en última instancia, una forma de conocimiento: conocimiento supremo de sí mismo y, a través de éste, conocimiento último de los secretos designios del universo: "Y como casi siempre sucede, la exploración de su universo habia sido, también lo empiezo a vislumbrar ahora, la exploración de mi propio y tenebroso mundo."21 En concreto, la visión final, implicada en la imagen Ojo Fosforecente, es la del tránsito de la ceguera a la videncia $y$, por ende, de la nada al todo, contradicciones que obviamente dejan de serlo en el reino del absoluto al que Fernando ha sido arrojado. ${ }^{22}$

\section{2) INTEGRACIÓN HOMBRE-UNIVERSO}

El "Informe sobre ciegos" objetiva, por lo tanto, la realidad absoluta que Fernando ha sido fatalmente arrastrado a develar. $Y$ si el trance del protagonista implica el conocimiento supremo de sí mismo a la vez que la comprensión de la verdad última del orbe, es dable concluir que con su experiencia Fernando ha trascendido la antinomia hombre-

Breton et les données fondamentales du surréalisme, Paris, 1950, p. 20). Mảs adelante añade, "il ne faudrait pas voir dans ce point suprême un simple point de vue thérique d'ou les contraires deviendraient conciliables, mais un point réel. . . situé à $\mathrm{l} a$ fois dans la réalité subjective de la conscience et dans l'univers extérieur." (p. 21).

21 Sobre béroes, p. 303.

22 Aunque Fernando emplea el calificativo de místico para autodefinirse la suya no es una experiencia mística en el sentido riguroso del vocablo; su visión participa de lo que se conoce como visión óffica. En un trabajo titulado precisamente The Orpbic Vision la autora, Gwendolyn Bays, traza las fronteras que delimitan ambos estados espirituales de enajenación: los receptores de la visión óffica - "nocturnal seers" - descienden a los infiernos del inconsciente pero no realizan la ascensión al cielo de la enajenación mística. Se trata de poetas como Arthur Rimbaud cuya obra la autora examina en su trabajo. También nos recuerda la señora Bays que para alcanzar tal cielo se requiere, "the thorny road of spiritual development". (The Orpbic Vision, Lincoln, Nebraska, 1964, p. 9), y luego agrega: "They (los receptores de la visión orfica) mistook their exploration of the unconscious for the mystical experience. Nevertheless, theirs was a task of vast importance, for the treacherous descent into Avernus, "the dark night of the soul', must precede the arduous ascent of Carmel which leads up the steep hill of self-naughting, nonattachment, and charity. ( $p, 30)$ En otro lugar hace hincapié en la oposición existente entre los símbolos con que los místicos y estos poetas objetivan sus experiencias: "Significantly, the seer poets chose the symbols water, darkness, and descent to describe their experiences, the exact opposites of those used by the mystics who spoke of theirs in terms of fire, light, and ascent." (p. 20) La autora también llama la atención al hecho de que la visión del infierno acarrea la locura, la destrucción y la muerte: "At length each of the seer poets arrived at the impasse of madness or the threat of madness" (p. 26), y luego agrega: "In one way or another each of these poets destroyed himself" (p. 29) Finalmente nos recuerda que en las profundidades del inconsciente, "the seer poets became aware of the primitive and the animal in themselves" (p. 25). 
universo. En rigor, esta antinomia constituye otra de las tantas contradicciones que los surrealistas han luchado por integrar invalidando la convicción de que hombre y cosmos pertenecen a dos órdenes diferentes y de que existe entre ellos un perpetuo estado de tensión. De hecho, con la vasta comprensión del todo - videncia-- quedan borradas las fronteras entre el ser y el orbe que éste habita.

No es todo, sin embargo. En el Informe la integración hombre-universo va también relacionada con lo que los surrealistas han llamado el azar objetivo, es decir, aquel lazo de unión, "qui existe entre l'automatisme subjectif personnel et l'automatisme universel. Ou si l'on préfère, entre l'inconscient personnel, l'inconscient collectif et même l'inconscient cosmique" ${ }^{23}$ la intrusión de lo maravilloso en la vida diaria, manifestada en coincidencias singulares, premoniciones o hechos no explicables por la razón, dan cuenta del azar objetivo. Ahora bien, la plasmación de esta noción en el Informe depende de la dicotomía deseocasualidad; es de notar que cada hecho, aparentemente fortuito en la vida de Fernando, no ha sido producto del azar sino que ha obedecido a una fuerza secreta que ha ido, a través de él mismo, forjando su destino puesto que, "cuando se movilizan no sólo las fuerzas conscientes de nuestra personalidad sino las más poderosas de nuestra subconsciencia, se termina por crear un campo de fuerzas telepáticas en torno de uno que impone a otros seres nuestra voluntad, y hasta se producen episodios que en apariencia son casuales pero que en rigor están determinados por esa invisible potencia de nuestro espíritu". ${ }^{24}$ No, no hay casualidades. Cada hecho en la vida de un ser se erige como la concretización de un deseo inconsciente $\mathrm{y}$, al mismo tiempo, cada deseo humano parece ser el reflejo del deseo divino o inconsciente cósmico, del destino o de la fatalidad de la que nadie escapa. A lo largo de su peregrinaje Fernando se cree guiado por la razón sólo para comprobar al final que la verdadera fuerza motriz han sido sus turbias obsesiones inconscientes.

Ahora bien, si el hombre impelido por sus profundas obsesiones engendra sus propias circunstancias y si éstas reflejan, de un misterioso modo, la voluntad divina, queda clara la correlación que se establece entre el orden divino y el humano. Para los surrealistas, sin embargo, el universo - regido por el azar- deviene así la expresión no de un orden sino del divino desorden: "The only God the Surrealists accept is their own image of the inconceivable.. The inconceivable, therefore, ac-

${ }^{23}$ Michel Carrouges, op. cit., p. 16.

24 Sobre béroes, p. 256. 
cording to the Surrealists, is disorder. Thus, disorder becomes the real god; and Providence which is generally revered as the manifestation of the mystical order of things, gives way to the worship of bazard, or chance, the mystical manifestation of the inconceivable but existent disorder of things."' 25

Fernando tiene plena conciencia de que cada acto de su vida y de los otros no es mera casualidad. En el momento de la iluminación final se le revela que, ". . . yo había sido llevado como un sonámbulo a aquellos dominios secretos de la Secta, pero también parecía como si durante años y años hubiese proyectado mis fuerzas más oscuras y profundas para convocar, finalmente, en aquel cuarto de Belgrano, a la mujer que en cierto modo más había deseado en mi vida." ${ }^{26}$ Con esta comprensión gradual del propio ser se le van también revelando los designios del universo: cuando el protagonista alcance el punto supremo de su visión se sabrá también reintegrado al orden o al desorden divino ya que, como afirma Carrouges: "L'homme marche en plein jour, au milieu d'un réseau de forces occultes qu'il lui suffirait de déceler et de capter pour s'avancer enfin victorieusement, à la face du monde, dans la direction du point suprême." ${ }^{27}$ Es obvio que Fernando no sólo ha captado sino que se ha integrado a las fuerzas ocultas que han moldeado su existencia. De esta manera y a medida que se acerca al momento supremo el protagonista siente que su ser, que ha estado siempre en peligro de desintegrarse, adquiere por fin consistencia, rea. lidad.

Fernando ha alcanzado el punto final de su viaje: su figura se erige como la de un formidable adversario, triunfante sobre la sociedad cosificada de la que él se ha arrancado y para la cual ha muerto. Rescatada su propia esencia, devuelto a la unidad primera, la ansiedad de $\operatorname{ser}^{28}$ - ser verdadera y auténticamente- de Fernando queda así rea-

25 Anna Balakian, op cit., p. 11. Por su parte, J. H. Matthews, otro estudioso del surrealismo, ha declarado que es precisamente en los hechos casuales e inesperados donde puede revelarse la clave para la comprensión de la existencia humana "Unexpected meetings, \curious coincidences, moments of prophetic insight... all these bear witness to the conviction that the key to human existence may be found, once man has learned how to search for it" An Introduction to Surrealish (Pennsylvania, 1965), p. 100.

28 Sobre béroes, p. 363.

27 Michel Carrouges, op. cit., p. 218.

28 En el "Informe sobre ciegos" Sábato hace mención de un caso muy comentado entre los surrealistas de París: el de Víctor Brauner, pintor que anticipó su propia ceguera en sus autorretratos. De acuerdo a la interpretación sabatiana, Brauner, movido por sus turbios deseos inconscientes, intercepta el vaso que otro pintor, Domínguez, ha arrojado y que no va dirigido a él: la pérdida de uno de los ojos materializa lo que Brauner había ya previsto $\longrightarrow$ deseado- en sus 
lizada. Y momentos antes de morir da cuenta de su liberación final: "Cosa singular: nadie ahora parece perseguirme". ${ }^{29}$

En concreto, la experiencia del autor del Informe se define, en última instancia, como una profunda experiencia espiritual y aun religiosa ya que el rescate del hombre que Sábato propone significa su salvación. Ha escrito Lionel Trilling respecto a la literatura de nuestros días: "more than with anything else, our literature is concerned with salvation. No literature has ever been so intensely spiritual as ours". ${ }^{30}$ En una época de crisis y de enjuiciamientos como la nuestra, en una época nihilista y sin fe, surge así una nueva perspectiva religiosa, profunda $y$ verdadera, de insospechada intensidad.

\section{CONCLUSIÓN}

En la persona de Fernando Vidal, Sábato ha objetivado su creencia de que sólo al artista le es dado rescatar la integridad del ser recuperando al mismo tiempo, la esencial unidad del universo. Es el artista quien trasciende la ceguera o mentira de nuestra civilización para alcanzar la verdad. Lo esencial, sin embargo, es que ésta solamente puede lograrse penetrando las fuerzas del mal: "El hombre real existe desde la caída. El hombre no existe sin el demonio: Dios no basta". ${ }^{31}$ La noción del mal en la obra de Sábato está entendida desde una doble perspectiva; por un lado apunta a la animalidad o fuerzas primarias que yacen latentes en el inconsciente individual y racial; por el otro, el mal es la ceguera en la que existe el hombre y en la que éste se ampara para perder conciencia de esas fuerzas infernales que sustentan su. vivir. Engañado -cegado- por un mundo aparencial en el que todo desde bancos y boutiques hasta bares y prostíbulos, contribuyen a sostenerlo, el ser ha velado la realidad subyacente, la esencia de la condición hu-

pinturas. El escritor Juan Larrea, por su parte, considera el caso Brauner como un hecho surrealista por excelencia en cuanto simboliza la adquisición de la anhelada Videncia. Opina Larrea: "En consecuencia, el hecho de conservar un ojo abierto a la realidad objetiva y mostrar el otro perforado afirmando simbólicamente $\mathrm{l}_{\mathrm{a}}$ visión introspectiva de una segunda realidad antinómica y comple. mentaria, figura una situación de síntesis entre ambas realidades, la objetiva y la subjetiva, la diurna y la nocturna, equivalente en símbolo a la adquisición de la tan suspirada Videncia." (Del surealismo a Macbupiccbu, México, 1967, p. 44); luego agrega, "el 'caso Brauner' ... constituye la realización simbólica de los deseos de ser del surrealismo" (p. 33 , el subrayado es nuestro). En concreto, en Fernando Vidal se realizan, en forma absoluta la Videncia y los anhelos de ser de los surrealistas.

29. Sobre béroes, p. 367.

30 Lionel Trilling, op. cit., p. 8.

31 Ernesto Sábato Gl escritor y sus fantasmas, p., 205. 
mana, el Mal, para seguir subsistiendo en aquel "Buenos Aires caótico de frenéticos muñecos con cuerda". ${ }^{32}$ En concreto, para Sábato cualquiera tentativa humana de salvación presupone una inmersión necesaria en los infiernos: sólo conociendo el mal, vale decir penetrando su esencia, puede el hombre atisbar algún tipo de verdad. Al artista le está destinada esta aventura; a él le toca llevar a cabo esta tarea de titanes que culmina, de algún modo, con su propia destrucción y que, en última instancia, lo convierte en figura redentora: "un gran artista", ha escrito Sábato, "es el hombre que tiene la facultad y la condena de levantar los velos que ocultan la terrible realidad a los simples mortales". ${ }^{33}$

El Informe constituye, en suma, el testimonio de un mundo rescatado en toda su compleja integridad. En él coexisten y se integran lo concreto y lo objetivo con el sueño, la alucinación, la locura. Las fuerzas demoníacas que lo sustentan van revelando un mundo extraño e incomprensible pero auténtico y real: tan auténtico y real como es Fernando, su protagonista. En la figura del héroe se integran el cielo y el infierno, la muerte y la vida, la fantasía y la realidad. Y en el Informe el autor Fernando-Sábato ha alcanzado aquel punto del que habló Bretón y donde, "la vida y la muerte, lo real y lo imaginario, lo pasado y lo futuro, lo comunicable y lo incomunicable, lo alto y lo bajo, dejan de ser percibidos como contradictorios". ${ }^{34}$

McGill University,

Z. Nelly Martínez

Montreal, Canada.

a2 Sobre béroes, p. 352.

33 Ernesto Sábato, Obras: Ensayos (Buenos Aires, 1970), p. 907.

84 A. Breton, op. cit., p. 75. 
